Cla esson: Neutr. u. saure Sulfate d. Methylalkohols. 231

welcher fast zwölf Mal so gross ist, als die von mir gefundene Menge ist wohl durch die Bestimmungsmethode bedingt worden.

Am Schlusse fühle ich mich gedrungen, dem Herm Prof. Dr. J. Rose, meinem hochverehrten Lehrer, für die Anleitung und für die vielfache Unterstützung bei dieser Arbeit meinen wärmsten Dank auszusprechen.

Strassburg im Elsass, März 1879.

\title{
Ueber die neutralen und sauren Sulfate des Methyl- and Aethylalkohols;
}

\author{
von \\ Peter Claesson.
}

Unter den Kohlenstoffrerbindungen sind die Aetherarten der Schwefelsäure früh Gegenstand eines eingehenden Studiums gewesen. Diese Untersuchungen haben sehr wichtige Beiträge geliefert zur jetzigen Auffassung der Constitution organischer Verbindungen.

Dessen ungeachtet hat unsere Kenntniss der genannten Aetherarten mit der Entwicklung unserer Kenntnisse der organischen Verbindungen in anderen Klassen nicht gleichen Schritt gehalten. So ist z. B. das neutrale Sulfat nur von einem einzigen Alkohol, dem Methylalkohol, mit Sicherheit bekannt. Während die sauren Sulfate (die Aetherschwefelsäuren) der einwerthigen Alkohole ziemlich vollständig studirt sind, hat unsere Kenntniss der entsprechenden Verbindungen der mehrwerthigen Alkohole eine geringere Bedeutung, weil sich aus ihrer Zusammensetzung nicht mit gleicher Sicherheit die Säurigkeit der Alkohole ergiebt, wie z. B. aus den Acetaten oder Nitraten. Was die Kohlenhydrate betrifft, so sind nur Versuche von Peligot bekannt, aus welchen nicht einmal mit Sicherheit hervorgeht, dass Dextrose mit Schwefelsäure unter Austritt von Wasser vereinigt werden kann. 


\section{Claesson: Ueber die neutralen u. sauren Sulfate}

Meine Untersuchungen haben zur Aufgabe gehabt, dieses Missverhältniss zu beseitigen, und, soweit möglich, war unsere Kenntniss der Sulfate von Alkoholen auf gleiche Stufe mit derjenigen der übrigen Aetherarten dieser Verbindungen zu bringen.

Um dieses Ziel zu erreichen, habe ich ein von der älteren Untersuchungsmethode, nämlich die resp. Alkohole und Schwefelsäure auf einander einwirken zu lassen, abweichendes Verfahren eingeschlagen.

Wie bekannt, ist die Einwirkung von Schwefelsäure, selbst auf die einwerthigèn Alkohole, nie so vollständig, dass sie der Reactionsgleichung $\mathrm{ROH}+\mathrm{H}_{2} \mathrm{SO}_{4}=\mathrm{HOSO}_{2} \mathrm{OR}$ $+\mathrm{H}_{2} \mathrm{O}$ entspricht, weil das bei der Reaction gebildete Wasser in einer entgegengesetzten Richtung wirkt, wie Berthelot unter Anderem hervorgehoben hat.

Um die Bildung von Wasser zu vermeiden, habe ich anstatt Schwefelsäure das $\mathbf{M o n o c h l o r i d ~ d i e s e r ~ S a ̈ u r e , ~} \mathrm{HOSO}_{2} \mathrm{Cl}$, in Anwendung gebracht. Unter der Voraussetzung nämlich, dass die Produkte bei der Einwirkung von Chlorsulfonsäure auf die Alkohole ausschliesslich, oder wenigstens hauptsächlich Aetherschwefelsäuren und Salzsäure sind, entsprechend der Gleichung $\mathrm{ROH}+\mathrm{HOSO}_{2} \mathrm{Cl}=\mathrm{HCl}+\mathrm{HOSO}_{2} \mathrm{OR}$, muss die Reaction so weit gehen, wie die Natur der Alkohole gestattet, und somit die Zusammensetzung der gebildeten Aethersäuren einen Rückschluss auf die Säurigkeit der Alkohole gestatten.

Wiewohl Chlorsulfonsäure weit energischer als Schwefelsäure auf die Alkohole wirkt, so ist doch die dabei statthabende Wärmeentwicklung nicht besonders gross, weil das bei der Reaction entwickelte Chlorwasserstoffgas einen grossen Theil derselben mit sich führt. Hieraus erklärt sich, dass diese Säure unter geeigneten Bedingungen auf die Alkohole, sowie auf die Kohlehydrate ohne Spur von Schwärzung oder tiefere Zersetzung wirkt. Es war dadurch möglich, die in chemischer Beziehung im Ganzen so wenig erforschten Kohlehydrate dem Kreise dieser Untersuchungen einzureihen, ein Gedanke, der das eigentliche Motiv zu denselben gewesen ist. 
Mehrere Chemiker haben schon die Einwirkung von Chlorsulfonsäure auf die einsäurigen Alkohole untersucht, aber ein nur flüchtiges Durchlesen ihrer Arbeiten zeigt die Nothwendigkeit einer Revision derselben. Ich werde sie im Nachstehenden durchgehen und kritisiren.

Die herrschende.Auffassung von der rationellen Zusammensetzung der Aetherschwefelsäuren und der damit im $\mathrm{Zu}$ sammenhang stehenden Frage von der gewöhnlichen Aetherbildung ist aus dem Streit hervorgegangen, welcher in der vorigen Hälfte unseres Jahrhunderts zwischen den Vertretern der verschiedenen Ansichten über die Constitution des Alkohols und Aethers, zwischen der Ammoniak-Aetherintheorie und der Ammonium-Aethyltheorie, zwischen Dumas, Berzelius und Liebig geführt wurde.

Es mag hier nur daran erinnert werden, dass unsere jetzige Auffassung sich auf A. Williams on's ${ }^{1}$ ) Untersuchung über Aetherbildung gründet. Er stellte gewöhnlichen Aether aus Natriumäthylat und Jodäthyl durch doppelte Zersetzung dar und zeigte, dass Aether Alkohol ist, in welchem ein Wasserstoffatom durch den Radical Aethyl ersetzt ist. Die bis dahin gewöhnliche Formel der Aetherschwefelsäure musste somit halbirt werden; letztere ist als "Alkoholsulfat" $\mathrm{C}_{2} \mathrm{H}_{5} \mathrm{SO}_{4} \mathrm{zu}$ betrachten. Durch Einwirkung dieser Verbindung auf Alkohol entsteht Aether und Schwefelsäure, welche letztere wieder auf den Alkohol reagirt. Hierdurch war diejenige Ansicht von der chemischen Constitution der Alkohole und einfachen Aetherarten festgestellt, welche heute noch gültig ist.

In dieser Abhandlung will ich meine Untersuchungen über die neutralen und sauren Sulfate (Aetherschwefelsäuren) des Methyl- und Aethylalkohols mittheilen. Die Abhandlung zerfällt in folgende Abtheilungen:

Geschichte und Kritik früherer Untersuchungen über die Eiıwirkung von Chlorsulfonsäure auf die einsäurigen Alkohole.

1) Ann. Chem. Pharm. 77, 37. 
234 Claesson: Ueber die neutralen $u$. sauren Sulfate

Ueber die Einwirkung von Chlorsulfonsäure auf Methylalkohol und die dabei entstehende Methylätherschwefelsäure.

Ueber Methylsulfat.

Ueber die Einwirkung von Chlorsulfonsäure auf Aethylalkohol und die dabei entstehende Aethylätherschwefelsäure.

Ueber die Menge der entstandenen Aetherschwefelsäure beim Mischen von Methyl- oder Aethylalkohol mit Schwefelsäure.

Ueber Chlorsulfonsäureäther und seine Einwirkung auf Alkohol.

Ueber eine neue Bildungsweise der Aethionsäure.

Ueber Aethylsulfat.

Ueber Gerhardt's Parathionsäure.

\section{Geschichte und Kritik früherer Untersuchungen über die Einwirknng von Chlorsulfonsänre anf die einwerthigen Alkohole.}

Baumstark ${ }^{1}$ ) untersuchte im Jahre 1866 die Einwirkung dieser Säure auf Alkohol, Aether und auf einige andere Körper. Die Säure reagirte sehr kräftig unter Schwärzung und theilweiser Zerstörung der organischen Substanz. Die bestimmbaren Verbindungen, welche Alkohol mit dieser Säure gab, sind nach ihm Aetherschwefelsäure und ein Oel, identisch mit Wetherill's schwefelsaurem Aethyl. Aether gab dieselben Verbindungen.

Dieser Abhandlung von Baumstark habe ich folgende Bemerkung hinzuzufügen.

Baumstark nimmt an, dass seine Säure Chlorsulfonsäure sei. Seine Analysen, wie auch eine Gasvolumbestimmung scheinen diese Annahme zu bestätigen. Aus seiner Beschreibung der Verbindung geht aber deutlich hervor, dass er sich hierin entschieden geirrt hat. Die Substanz, mit welcher er operirte, war wahrscheinlich Pyrosulfurylchlorid,

1) Ann. Chem. Pharm. 140, 78. 
durch bedeutende Mengen von Phosphortrichlorid oder Phosphoroxychlorid verunreinigt. Seine Säure war nämlich, sagt er selbst, eine ölartige, schwach gelbe Flüssigkeit, die in Wasser getröpfelt zu Boden sank und allmählich in Schwefelsäure und Salzsäure zersetzt wurde: Eigenschaften, welche nach Michaelis ${ }^{1}$ ) dem Pyrosulfurylchlorid zukommen, was auch von mir bestätigt werden kann. Dass Baumstark's Säure Pyrosulfurylchlorid war, lässt sich weiter aus der Gasvolumbestimmung, welche derselbe damit ausfuhrte, schliessen. Diese stimmt zwar ganz gut mit der für Chlorstlfonsäure berechneten Zahl; aber die Chlorsulfonsäure in Gasform ist nach Williams ${ }^{2}$ ) grösstentheils in Schwefelsäureanhydrid und Chlorwasserstoff dissociirt. Baumstark's Bestimmung wäre ganz unerklärlich, wenn seine Verbindung nicht ein stark verunreinigtes Pyrosulfurylchlorid gewesen wäre. ${ }^{3}$ ) Es geht somit hieraus hervor, dass Baumstark's

1) Jen. Zeitschr. 6, 235 und 292.

2) Zeitschr. Chem. 1869.

3) Die Chlorsulfonsäure habe ich nach Michaelis dadurch hergestellt, dass Phosphorpentachlorid zur schwach rauchenden Schwefelsäure zugesetzt wurde, gemäss dem Verhältniss: $\mathrm{PCl}_{5}+\mathrm{H}_{2} \mathrm{SO}_{4}$. Hiernach destillirte ich aus einer untubulirten Retorte, und fing das zuerst Uebergehende, hauptsächlich aus Phosphortrichlorid bestehend, besonders auf, bis eine Probe des Destillates unter starker Explosion auf Wasser einwirkte, ohne dass zuvor Deltropfen sich absonderten, worauf die farblose Chlorsulfonsäure übergeht. In der Retorte bleibt Metaphosphorsäure und etwas Schwefelsäure zurück. Die so erhaltene Säure ist jedoch nicht ganz rein, namentlich enthält sie etwas Phosphorverbindungen und freie Schwefelsäure. Auch wurde die Säure dargestellt aus Chlorwasserstoff und Schwefelsäureanhydrid, oder, was dasselbe ist, durch Einleiten von Chlorwasserstoff in rauchende Schwefelsäure: eine gute Methode, wenn anhydridreiche Säure zur Verfügung steht.

Baumstark gewann seine Säure dadurch, dass er Phosphorpentachlorid zu rauchender Schwefelsäure setzte, bis die gebildete obere Schicht $2 / 3$ der unteren ausmachte, worauf destillirt wurde. Eine solehe Theilung in awei Sehichten tritt aber nur dann ein, wenn während der Operation nicht geschüttelt wird. Auch hat Baumstark zu viel Phosphorpentachlorid angewendet, wodurch die zunächst entstandene Chlorsulfonsäure in Pyrosulfurylchlorid übergegangen ist. Phosphorpentachlorid und Chlorsulfonsäure geben nämlich nach Michaelis Pyrosulfurylchlorid und Phosphoroxychlorid. 
Untersuchung keine Bedeutung hat für die Frage, welche hier erörtert werden soll.

Im Jahre 1875 publicirte Marjan Orlowsky ${ }^{1}$ ) eine vorläufige Notiz über die Einwirkung von Chlorsulfonsäure auf Aethyl-' und einige andere Alkohole. Er giebt an, dass dabei Sulfate gebildet werden laut der Formel: $\mathrm{HOSO}_{2} \mathrm{Cl}$ $+2 \mathrm{C}_{2} \mathrm{H}_{5} \mathrm{OH}=\left(\mathrm{C}_{2} \mathrm{H}_{5}\right)_{2} \mathrm{SO}_{4}+\mathrm{HCl}+\mathrm{H}_{2} \mathrm{O}$. Auch Phenol gab eine analoge Verbindung von der Zusammensetzung $\left(\mathrm{C}_{6} \mathrm{H}_{5}\right)_{2} \mathrm{SO}_{4}$. Diese Sulfate hatten jedoch ganz andere Eigenschaften, als Dumas' Methylsulfat oder W etherill's Aethylsulfat. Sie waren neutral reagirende, undestillirbare Flüssigkeiten, die von Wasser augenblicklich in Aetherschwefelsäuren und Alkohol zersetzt wurden. Sie waren darnach mit der Wetherill'schen Verbindung isomer. Diese letztere wurde als der Aether der Isäthionsäure angesehen.

Diese Annahmen wurden etwas später ausführlicher entwickelt in einer Abhandlung, offenbar von derselben Person (die völlig übereinstimmenden analytischen Angaben beweisen dieses), welche doch den Namen eines anderen Verfassers trägt, nämlich Marja Mazurowska. ${ }^{2}$ ) Mazurowska unterscheidet sich nur in einem Punkte von ihrem Synonym Orlowsky, dessen Notiz sie übrigens weder citirt, noch erwähnt. Sie hat nämlich gefunden, dass Chlorsulfonsäure sich nicht auf gleiche Weise zu Phenol, wie zu den Alkoholen der Fettreihe verhält. Das Produkt aus der Einwirkung von Chlorsulfonsäure auf Phenol hat freilich ganz dieselben Eigenschaften, sagt sie, wie die Produkte aus den Fettalkoholen und derselben Säure; aber mit Wasser verdünnt, neutralisirt und mit Kaliumsulf hydrat destillirt, wurde "wider Erwarten" keine Spur von Phenolmercaptan gewonnen. In der von Orlowsky veröffentlichten Notiz wird aber angegeben, dass das Produkt dabei "den nicht verkennbaren Geruch von Phenolmercaptan ausstösst". Mazurowska fand weiter, dass das von ihr untersuchte Kalisalz grosse Aehnlichkeit mit dem Salz von Paraphenolsulfonsäure habe. Sie betrach-

1) Ber. Berl. chem. Gres. 8, 332.

2) Dies. Journ. [2] 13, 158 . 
tete das Produkt aus Chlorsulfonsäure und Phenol als paraphenolsulfonsauren Phenoläther. Orlowsky dagegen "freut sich, die von Kekulé ausgesprochene Isomerie unter den Phenolschwefelsäuren durch die Darstellung der wirklichen Phenolschwefelsäure zum Abschluss gebracht zu haben". Wie man sieht, fügen sich die Resultate der Experimente genau den nachstehenden Auffassungen des Experimentators. Vielleicht hat Orlowsky-Mazurowska's Reactionsgleichung $2 \mathrm{ROH}+\mathrm{HOSO}_{2} \mathrm{Cl}=\mathrm{R}_{2} \mathrm{O}_{2} \mathrm{SO}_{2}+\mathrm{HCl}+\mathrm{H}_{2} \mathrm{O}$ nur eine solche subjective Wirklichkeit.

Wir wollen darum die Versuche etwas näher betrachten, aus welchen, wie Mazurowska sagt, mit Nothwendigkeit hervorgeht, dass die von ihr hergestellten Körper die neutralen Aetherarten der Schwefelsäure sind.

Mazurowska tröpfelte 1 äq. Chlorsulfonsäure zu 2 äq. absolutem Alkohol. „Dämpfe von Wasser und Salzsäure“ entwickelten sich dabei, und es blieb eine undestillirbare Flüssigkeit zurück, die sich völlig nentral zeigte, aber bei Zusatz von Wasser momentan in Aetherschwefelsäture und Alkohol zersetzt wurde. Onlöslich in Aether, wurde das Oel damit von überschüssigem Alkohol befreit und schliesslich über Schwefelsäure getrocknet.

Das so aus Aethylalkohol gewonnene Produkt, das einzige, welches analysirt wurde, gab bei der Analyse folgende Zahlen:

$\begin{array}{ccc}\text { Für Aethylsulfat berechnet. } & \text { Erhalten, } \\ \text { C } & \mathbf{3 1 , 1 6} & 31,16 \\ \text { H } & \mathbf{6 , 4 6} & 6,53 \\ \text { S } & 20,77 & 21,15\end{array}$

Wie man sieht, entsprechen die Resultate der Analyse, wie Mazurowska naiv sagt, „mit hinlänglicher Genauigkeit" den für das neutrale Sulfat berechneten Werthen.

Es muss aber geprüft werden, wie es sich mit der Garantie für die Reinheit der analysirten Produkte verhält.

Ganz unerklärlich bleibt es, wie das weggehende Chlorwasserstoffgas das gebildete Wasser mit sich nehmen kann, besonders wenn man bedenkt, dass der Alkohol eine grosse Verwandtschaft zum Wasser hat, was auch der Fall mit dem 
Produkt selbst ist, welches sich bei Zusatz von Wasser stark erhitzt. Die „Dämpfe von Wasser und Salzsäure" könnten darum vielleicht sich nur aus der gewöhnlichen Erscheinung herleiten, welche immer eintritt, wenn zwei Flüssigkeiten unter heftiger Gasentwicklung gemischt werden, nämlich dass das entwickelte Gas Partikelchen von der Flüssigkeit mechanisch mit sich führt.

Weiter lässt sich auch schwierig einsehen, dass Mazurowska's Produkt ein neutrales Sulfat gewesen sei, weil, wie Mazurowska selbst sagt, Wasser augenblicklich die Sulfate zersetzt, und zwei Substanzen, die einander zersetzen, nicht beide Endprodukte sein können. Ein Theil des Produkts muss somit nothwendig eine Aetherschwefelsäure sein.

Es ist ferner sehr auffallend, dass Mazurowska's Verbindungen als neutrale Sulfate in Aethyläther unlöslich sind. Diesen Umstand benutzt aber Mazurowska dazu, das Produkt zu reinigen. Wie viel reiner es dadurch wurde, lässt sich leicht aus Folgendem beurtheilen. Mazurowska's Produkt ist nicht, wie sie angiebt, in Aether unlöslich, sondern wirklich löslich, wenn man, wohl zu merken, wasserfreien Aether anwendet. In gewöhnlichem wasserhaltigem Aether ist die Substanz dagegen "wirklich, wie Mazurowska angiebt, unlöslich, was sicherlich darauf beruht, dass das Produkt Wasser aus dem Aether aufnimmt und damit eine in Aether unlösliche Verbindung eingeht.

Aus der Abhandlung Mazurowska's geht aber hervor, dass es ausser der Analyse eigentlich die neutrale Reaction gewesen, welche das sicherste Kennzeichen ausmachte, dass die Produkte als wirklich neutrale Aetherarten der Schwefelsäure zu betrachten wären. Mazurowska war somit die Thatsache unbekannt, dass das Reactionspapier seinem gewöhnlichen Farbenwechsel, in Berührung mit wasserfreien Substanzen, nicht unterworfen ist, diese mögen nun Säuren oder Alkalien sein. Die neutrale Reaction beweist somit gar nichts.

Es giebt aber viele andere Eigenthümlichkeiten in $\mathrm{Ma}$ zurowska's Abhandlung, ja so viele, dass ich behaupten 
kann, dass kein einziger Satz darin vollständig wahr ist. Ich werde hier, nur einen oder ein paar hervorheben.

Mazurowska zersetzt ihr neutrales Aethylsulfat mit Wasser, neutralisirt mit kohlensaurem Baryt und erhält beim Abdampfen Krystalle von ätherschwefelsaurem Baryt. Eine Bariumbestimmung giebt, wie beinahe alle ihre Analysen, Resultate, die auf $1 / 100$ pCt. mit den berechneten übereinstimmen, aber merkwürdiger Weise sind nicht blos dieses, sondern alle ihre ätherschwefelsauren Barytsalze vom Methyl bis auf Amyl wasserfrei, während alle Anderen sie wasserhaltig gefunden haben.

Auch ihre Deutung der Arbeiten ihrer Vorgänger ist in hohem Grade merkwürdig. Ueber die Untersuchung Müller's ${ }^{1}$ ) heisst es:

"Auch die Resultate, welche er bei Einwirkung von Sulfuryloxychlorid auf Alkohol erhalten hatte, erwähnt er blos oberflächlich, bemerkt indess, dass er durch Modification des Verfahrens und der Verhältnisse auch nach der Methode von Baumstark nebenbei den isomeren Oxyäthansulfosäureäthyläther $\mathrm{C}_{2} \mathrm{H}_{4} \mathrm{SO}_{2} \mathrm{OH}_{2} \mathrm{OH}_{5}$ erhalten habe."

Müller sagt jedoch ausdrücklich in der citirten Arbeit, dass er den Aether der Isäthionsäure nicht habe erhalten können, weder direct aus der Säure, noch auf eine andere Weise. Ja, er erwähnt gar keines Versuches mit Alkohol und Chlorsulfonsäure. Nur einen Versuch mit Methylalkohol citirt er und erhält dabei Methylätherschwefelsäure und Chlorwasserstoff.

Mazurowska erwähnt mit keinem Wort der Isomerie zwischen ihrem und Dumas' Methylsulfat.' Diese wäre auch schwer zu erklären, da ja, wie bekannt, die Gesammtmenge des Schwefels in Dumas' Aether beim Kochen mit Wasser in Schwefelsäure übergeht, ein Beweis, dass die Verbindung wirklich als Sulfat des Methylalkohols angesehen werden muss. Es giebt, wie gesagt, manche andere Fehler in Orlowsky-Mazurowska's Abhandlungen, aber die angeführten

1) Inalugural-Dissertation, Göttingen 1874. 
240 Claesson: Ueber die neutralen u. sauren Sulfate mögen genügen, um zu zeigen, wie wenig diese Arbeiten geeignet sind, die behandelte Frage ihrem Ziele näher zu führen.

\section{Ueber die Einwirkung von Chlorsulfonsäure auf Methylalkohol und die dabei entstehende Methyl- ätherschwefelsäure.}

Gleiche Moleküle absoluten Methylalkohols (von Kahlbaum's Fabrik, noch einmal über gebranntem Kalk rectificirt) und Chlorsulfonsäure wurden auf solche Weise gemengt, dass der Alkohol mittelst eines Scheidetrichters langsam in die Säure eintropfte. Die Säure wurde während der Operation mit Eis abgekühlt. Die Mischungsretorte wurde mit einer leeren Vorlage versehen, deren Tubulus mit einem gewogenen Chlorcalciumrohr, mit Schwefelsäure befeuchtete Bimssteinstïcke enthaltend, verbunden war, worauf eine mit Wasser gefüllte und danach gewogene W oulff'sche Flasche folgte, deren anderer Tubulus mit einem Aspirator verbunden war. Der Zweck dieses Apparates war, zu bestimmen, ob das weggehende Chlorwasserstoffgas Wasser mit sich führt, wie Mazurowska angiebt. Jedem Tropfen Alkohol folgte eine heftige Chlorwasserstoffentwicklung, die während der ganzen Operation fortdauerte. Schliesslich wurde das in der Mischung gelöst enthaltene Chlorwasserstoffgas durch gelindes: Erhitzen im Vacuum grösstentheils ausgetrieben.

Von 28 Grm. Methylalkohol und 100 Grm. Chlorsulfonsäure wurden $100 \mathrm{Grm}$. von der Mischung und $29 \mathrm{Grm}$. Chlorwasserstoffgas erhalten. Das Chlorcalciumrohr hatte nur 0,5 Grm. an Gewicht zugenommen, was von den kleinen Partikeln der Flüssigkeit herrührte, die von der heftigen Gasentwicklung mitgeführt wurden.

Die Uebereinstimmung der erhaltenen mit den laut der Gleichung $\mathrm{HOSO} 2 \mathrm{Cl}+\mathrm{CH}_{3} \mathrm{OH}=\mathrm{HOSO}_{2} \mathrm{OCH}_{3}+\mathrm{HCl}$ berechneten Werthen zeigt folgende Zusammenstellung:

Berechnet. Gefunden.

$\begin{array}{llcl}\mathrm{HCl} & 31 & \mathrm{Grm} . & 29 \mathrm{Grm} . \\ \mathrm{HOSO}_{2} \mathrm{OCH}_{3} & 98 & & \end{array}$


Die analytischen Resultate des so erhaltenen Produkts sind mit den für Methylätherschwefelsäure berechneten Werthen zusammengestellt:

$\begin{array}{ccc} & \text { Berechnet. } & \text { Gefunden. } \\ \text { C } & 10,71 & 9,32 \\ \text { H } & 3,57 & 3,84 \\ \text { S } & 28,57 & 26,85\end{array}$

Das Atomgewichtsverhältniss zwischen den gefundenen Werthen von Kohlenstoff und Schwefel sind 1 C:1,1 S. Zugleich enthielt das Produkt ungefähr 2 pCt. Chlor und Spuren von Phosphor.

Das analysirte Produkt wurde mit Wasser versetzt, wobei eine geringe Trübung und ein stechender Geruch hervortritt, ohne Zweifel von dem Methyläther der Chlorsulfonsäure berrührend, mit kohlensaurem Kali neutralisirt, darauf zur Trockne im Wasserbade verdunstet, und die trockne Masse mit warmem Alkohol extrahirt. Aus der alkoholischen Lösung krystallisirt methylätherschwefelsaures Kali in fettigen Krystallmassen; was Zusammensetzung und iibrige Eigenschaften betrifft, so stimmt es mit dem aus Methylalkohol und Schwefelsäure erhaltenen überein.

Beim Erhitzen des obigen Produkts im Vacuum bei $130^{\circ}-140^{\circ}$ wird es beinahe quantitativ laut der Formel:

$$
2 \mathrm{HOSO}_{2} \mathrm{OCH}_{3}=\left(\mathrm{CH}_{3}\right)_{2} \mathrm{O}_{2} \mathrm{SO}_{2}+\mathrm{H}_{2} \mathrm{SO}_{4}
$$

zersetzt, somit in Methylsulfat, das langsam überdestillirt, und Schwefelsäure, die in der Retorte zurückbleibt. Von $28 \mathrm{Grm}$. der erwähnten Mischung wurden 13,5 Grm. Methylsulfat erhalten, während unter Voraussetzung, dass das Produkt reine Methylätherschwefelsäure und die Umsetzung vollständig wäre, 15,7 Grm. Methylsulfat erhalten werden sollten.

Das analysirte Produkt war somit Methylätherschwefelsäure mit etwa $10 \mathrm{pCt}$. Verunreinigungen, hauptsächlich aus Schwefelsäure, Salzsäure, Phosphorsäure uud chlorsulfonsaurem Methyläther bestehend. Mazurowska's Produkt war demnach Methylätherschwefelsäure mit denselben Verunreinigungen, wozu noch kamen: Wasser von dem zugesetzten wasserhaltigen Aether und wenigstens ein Theil von dem zweiten Molekül Alkohol, das in Anwendung gebracht war. 


\section{Claesson: Ueber die neutralen u. sauren Sulfate}

Die wasserfreie Methylätherschwefelsäure ist eine durch die heftige Reaction etwas dunkel gefärbte, ölartige Flüssigkeit, die an Glas nicht adbärirt und durch Abkühlung bis auf $-30^{n}$ nicht fest wird. In wasserfreiem Aether ist sie in allen Verhältnissen löslich; wird aber gewöhnlicher wasserhaltiger Aether in hinreichender Menge zugesetzt, so trennt sich die Säure von dem Aether, was darauf beruht, dass die Aethersäure Wasser aus dem Aether aufnimmt und damit eine Verbindung eingeht, die in Aether unlöslich ist. Die Methylätherschwefelsäure bindet somit moleculares Wasser. Die Erhitzung bei der Verdünnung der Säure mit Wasser ist sehr stark. Auch tritt eine schwache Erwärmung ein beim Zusatz von Methylalkohol, und bei einer darauf folgenden Destillation gehen erst über $100^{\circ}$ Methyläther, Methylalkohol und schliesslich Methylsulfat über. Die Aethersäure bindet somit auch Methylalkohol molecular. Das Verhalten beim Erhitzen habe ich schon erwähnt.

Bekanntlich erhielten Dumas und Peligot ${ }^{3}$ ) Methylätherschwefelsäure in krystallisirtem Zustande. Thre Angabe darüber lautet folgendermaassen:

"Quand on mélange de l'acide sulfurique concentré et de l'esprit de bois, il se dégage beaucoup de chaleur, et il se forme une grande quantité de bisulfate de méthylène; quelquefois même par l'evaporation spontanée du mélange nous avons obtenu celui-ci à l'état cristallisé; mais il nous a été impossible de reproduire cette cristallisation à volonté.

Il est très facile au contraire de se procurer le bisulfate de méthylène au moyen du sulfate double de méthylène et de baryte. Ce sel étant dissous dans l'eau, on y ajoute peu à peu assez d'acide sulfurique pour precipiter la baryte d'une manière exacte, on filtre et on évapore la liqueur dans le vide. Quand elle a acquis l'état sirupeux, le bisulfate de méthylène cristallise en aiguilles blanches. Ce corps est très altérable dans le vide, il se détruit promptement en formant du gaz sulfureux."

Hieran knüpfe ich folgende Bemerkung. Weil die

1) Ann. chim. phys. 58, 54 . 
wasserfreie Säure nicht einmal bei $-30^{\circ}$ fest wird, so muss die von Dumas und Peligot gewonnene krystallisirte Säure Krystallwasser enthalten. Ich habe darum versncht, die Säure krystallisirt zu erhalten, indem ich in verschiedenen Versuchen 1, 2 und 3 Aeq. Wasser zu der wasserfreien Säure setzte und danach im Vacuum verdunstete. Aber es ist mir noch nicht geglückt, weder dadurch, noch durch die Methode Dumas', die Säure krystallisirt zu erhalten. Ihre Angabe, dass die krystallisirte Säure schon bei gewöhnlicher Temperatur unter Bildung von schwefliger Säure zerstört wird, ist unstreitig geeignet, $Z$ weifel einzuflössen, in wie fern die krystallisirte Substanz, die diese berühmten Chemiker erwähnen, wirklich Methylätherschwefelsäure gewesen sei, besonders da keine Analyse vorgenommen wurde. In wasserfreiem Zustande kann die Süure weit über $100^{\circ}$ erhitzt werden ohne Bildung von schwefliger Säure, und in wasserhaltigem Zustande ist sie bekanntlich einer ganz anderen Zersetzung unterworfen. Ich muss daher behaupten, dass die Methylätherschwefelsäure nicht krystallisirt werden kann.

Schliesslich will ich hier erwähnen, dass die Reaction zwischen Chlorsulfonsäure und Methylalkohol dieselbe ist, wenn die oben beschriebene Methode umgekehrt wird, derart, dass die Säure vorsichtig zu dem Alkohol gesetzt wird. Auch hier reagiren die beiden Körper zu gleichen Molekülen auf einander, unter Bildung von Methylätherschwefelsäure und Salzsäure. Die Reaction ist jedoch hier nicht so glatt, weil der entstehende Chlorwasserstoff im Anfange von dem Alkohol aufgenommen wird und sich somit theilweise in $\mathrm{Me}$ thylchlorid und Wasser umsetzt, welche letztere Verbindung mit der Chlorsulfonsäure Chlorwasserstoff und freie Schwefelsäure bildet.

\section{Ueber Methylsulfat.}

Wie oben erwähnt, wird die wasserfreie Methylätherschwefelsäure beim Erhitzen auf $130^{\circ}-140^{\circ}$ in Methylsulfat und Schwefelsäure zerlegt. Schon bei niedrigerer Temperatur fängt die Zersetzung an, geht dann aber sehr langsam 


\section{Claesson: Ueber die neutralen $\mathfrak{u}$. sauren Sulfate}

weiter. Die Operation wurde in einer Retorte mit luftdicht schliessender Vorlage vorgenommen. Nach dem Evacuiren geht das Sulfat beim Erhitzen der Aethersäure auf die genannte Temperatur langsam über. Das überdestillirte Sulfat wurde nochmals im Vacuum rectificirt, wonach es vollständig rein ist. So dargestellt, stimmt es in allen wesentlicheu Eigenschaften mit dem nach der Methode von Dumas erhaltenen überein: Siedepunkt (Säule vollständig im Dampf) $187^{\circ}-188^{\circ}$, spec. Gew. bei $18^{\circ} 1,327$. Dumas giebt den Siedepunkt $188^{\circ}$ und das spec. Gew. 1,324 bei $22^{\circ}$. Bekanntlich stellten Dumas und Peligot ${ }^{1}$ ) diese Verbindung durch vorsichtiges Destilliren von 1 Thl. Methylalkohol und 8-10 Thln. Schwefelsäure her. Ich habe diesen Versuch wfederholt und kann seine Richtigkeit im Wesentlichen bestätigen.

Sie behaupten, dass das Methylsulfat zwiebelähnlich rieche. Das von mir dargestellte Methylsulfat hat im Gegentheil einen angenehmen und erfrischenden, etwas an Pfeffermünze erinnernden Geruch. Der zwiebelähnliche Geruch muss somit von Verunreinigungen des Methylalkohols hergerührt haben.

Weiter wird angegeben, dass das Methylsulfat ohne Zersetzung bei gewöhnlichem Luftdruck siedet. Es ist aber leicht wahrzunehmen, dass bei wiederholtem Destilliren Zersetzung eintritt, die beim Sieden mit anfwärts gerichtetem Kühler bald sehr bedeutend wird. Im Vacuum siedet es aber völlig unzersetzt.

Dumas und Peligot geben weiter an, dass das Gewicht des erhaltenen Methylsulfats eben so gross sei, wie das der angewendeten Menge von Methylalkohol. Ich muss hierin einen Schreibfehler sehen. Das Gewicht des ganzen Destillates ist allerdings so gross wie das des angewendeten Alkohols, aber das Destillat besteht zum grössten Theil aus Wasser, entstanden durch die Oxydation von Methylalkohol durch Schwefelsäure. Wie vorsichtig auch die Destillation vorgenommen wird, so wird der grösste Theil des Methylalkohols zu humusartigen Substanzen oxydirt, unter Ent-

1) Ann. chim. phys. 58, 万4. 
wicklung von schwefliger Säure. Die Ausbeute an Methylsulfat ist in der That sehr gering. Auch braucht man nicht einen solchen Ueberschuss von Schwefelsätre anzuwenden, wie Dumas und Peligot angeben. Die Ausbeute wird nicht geringer, wenn Methylalkohol und Schwefelsäure im Verhältniss von gleichen Molekülen destillirt werden.

\section{Ueber die Einwirkung von Chlorsulfonsäure auf Aethylalkohol und die dabei entstehende Aethyl- ätherschwefelsänre.}

Wie sich aus obigen Versuchen leicht schliessen lässt, verhält sich Aethylalkohol zur Chlorsulfonsäure in der Hauptsache ebenso, wie Methylalkohol. Wenn unter denselben Verhältnissen operirt wird, wie bei Methylalkohol oben angegeben wurde, so ist das Hauptprodukt immer Aethylätherschwefelsäure und Chlorwasserstoff.

Der Gang der Reaction variirt aber hier etwas mehr als beim Methylalkohol, je nach der Temperatur und je nachdem Alkohol oder Säure bei der Operation im Ueberschuss vorhanden ist. Lässt man unter guter Kühlung den Alkohol langsam zu der Säure tropfen, so bildet sich beinahe nur Chlorwasserstoff und Aethylätherschwefelsäure. Das in Wasser gebrachte Produkt scheidet nur sehr wenig von dem Chlorsulfonsäureäthyläther ab. Wird aber der Mischungskolben während der Operation nicht abgekiühlt, so entsteht Chlorsulfonsäureäther in ziemlich bedeutender Menge. Die Reaction vollzieht sich somit auch hier in zwei Richtungen nach den Gleichungen:

1) $\mathrm{C}_{2} \mathrm{H}_{5} \mathrm{OH}+\mathrm{HOSO}_{2} \mathrm{Cl}=\mathrm{HOSO}_{2} \mathrm{OC}_{2} \mathrm{H}_{5}+\mathrm{HCl}$,

2) $\mathrm{C}_{2} \mathrm{H}_{5} \mathrm{OH}+\mathrm{HOSO}_{2} \mathrm{Cl}=\mathrm{C}_{2} \mathrm{H}_{5} \mathrm{OSO}_{2} \mathrm{Cl}+\mathrm{H}_{2} \mathrm{O}$,

und das Verhältniss zwischen den beiden Reactionen variirt je nach der Temperatur. Ist der Alkohol im Ueberschuss, so entsteht hier, wie beim Methylalkohol, durch die Einwirkung von Chlorwasserstoff auf Alkohol Chloräthyl und somit eine entsprechende Menge freier Schwefelsäure

Die Eigenschaften der erhaltenen Aethylätherschwefelsäure gleichen vollständig denen der Methylätherschwefel- 
246 Claesson: Ueber die neutralen u. sauren Sulfate

säure. Nur ist beim Erhitzen die Zersetzung in Aethylsulfat und Schwefelsäure weit weniger glatt. Die Masse verkohlt beim Erhitzen zum grössten Theil unter Entwicklung von schwefliger Säure.

\section{Ueber die Menge der entstandenen Aetherschwefel- säure beim Vermischen von Methyl- oder Aethyl- alkohol mit concentrirter Schwefelsäure.}

Es herrschen in der chemischen Literatur verschiedene Meinungen darüber, wie viel Aetherschwefelsäure in einem Gemenge von Alkohol und Schwefelsäure gebildet wird. $\mathrm{Hennel}^{1}$ ), welcher gleiche Gewichtstheile Schwefelsäure und Alkohol von $90 \mathrm{pCt}$. anwendete, giebt an, dass $56 \mathrm{pCt}$. Aetherschwefelsäure gebildet werden. Millons ${ }^{2}$ ) Angabe lautet dahin, dass unter Anwendung von gleichen Aequivalenten Alkohol und Schwefelsäure unter $0^{\circ}$ keine Aetherschwefelsäure gebildet wird, dass aber schon bei $15^{0} 77 \mathrm{pCt}$. derselben entstehen, ein Gehalt, der nicht überstiegen werden konnte. Schliesslich theilt Berthelot ${ }^{3}$ ) mit, dass unter Anwendung von gleichen Molekülen $59 \mathrm{pCt}$. Aetherschwefelsäure als Maximum gebildet werden. Berthelot fand für folgende beiden Mischungen:

1) $\mathrm{H}_{2} \mathrm{SO}_{4}+\mathrm{C}_{2} \mathrm{H}_{5} \mathrm{OH}+1 / 3 \mathrm{H}_{2} \mathrm{O}$,

2) $\mathrm{H}_{2} \mathrm{SO}_{4}+\mathrm{C}_{2} \mathrm{H}_{5} \mathrm{OH}+11 / 3 \mathrm{H}_{2} \mathrm{O}$,

die folgenden Grenzwerthe:

$\begin{array}{ccccc} & 40 \mathrm{Stdn} . & 90 \mathrm{Stdn} . & 20 \text { Tage. } & \mathbf{1 4 7} \text { Tage. } \\ \mathbf{1}) & \mathbf{5 6 , 0} & \mathbf{5 7 , 4} & 59,0 & 58,8 \\ 2) & \mathbf{1 3 , 2} & 21,2 & 41,2 & 54,8\end{array}$

Alkohol mit 25 pCt. Wasser gab nach Ablauf von einem Monat nur 8 pCt. Aetherschwefelsäure.

Zur Prüfung der verschiedenen Angaben habe ich einige Controlbestimmungen gemacht, die ich hier mittheilen will.

Absoluter Alkohol und reines Schwefelsäurehydrat wur-

1) Ann. ehim. phys. (1829) 42, 77.

2) Daselbst (1847) 19, 227.

3) Bull. Soc. Chim. (1873) 19, 295. 
den unter Anwendung von $1 \mathrm{Mol}$. Schwefelsäure (50 Grm.) auf resp. $1 / 2,1,1 \frac{1}{2}, 2,2^{1} / 2,3$ und 4 Mol. Alkohol gemengt. Die Mischung wurde $1 / 4$ Stunde auf dem Wasserbade erwärmt, dann nach dem Erkalten erst mit Eis und nachher mit Wasser auf $500 \mathrm{Cc}$. verdünnt, wonach diese Lösung titrirt wurde. Folgende Zahlen drücken für die oben stehenden Mischungen in Procenten den Gehalt an erzeugter Aetherschwefelsäure aus.

$$
\begin{array}{ccccccc}
1 / 2 & 1 & 11 / 2 & 2 & 21 / 2 & 3 & 4 \\
73,1 & 57,1 & 59,6 & 65 & 72 & 77,4 & 77,4
\end{array}
$$

Hieraus geht hervor, dass unter Anwendung von gleichen Molekülen Alkohol und Säure 57,1 pCt. Aetherschwefelsäure gebildet werden, aber dass diese Ausbeute durch erhöhten Zusatz entweder von Alkohol oder Schwefelsäure gesteigert wird. Unter Anwendung von $3 \mathrm{Mol}$. Alkohol auf $1 \mathrm{Mol}$. Schwefelsäure ist das Maximum 77,4 pCt. erreicht.

Bei einer anderen ähnlichen Mischung von gleichen Molekülen Alkohol und Schwefelsäure wurde nach dem Neutralisiren mit Kalihydrat der Gehalt an der vollständig an Kali gebundenen Schwefelsäure in gewöhnlicher Weise bestimmt. Hieraus berechnet sich die Menge der Aetherschwefelsäure zu $54 \mathrm{pCt}$.

Ein dritter Versuch ergab Folgendes: Die Mischung wurde nach Verdünnen mit Wasser durch Zusatz von Kalihydrat neutralisirt, zur Trockne verdunstet und mit kochendem Alkohol extrahirt. Aus dem Gewichte des in Lösung übergegangenen und trocken gewogenen Salzes lässt sich berechnen, dass $52 \mathrm{pCt}$. Aetherschwefelsäure erzeugt sind.

Die Ergebnisse dieser Versuche stimmen somit ganz nahe mit Hennel's und Berthelot's Resultaten überein. Millon's Angabe muss somit fehlerhaft sein, was um so wahrscheinlicher ist, als seine andere Angabe, dass 1 Aeq. Schwefelsäure weniger Aetherschwefelsäure gebe, wenn sie mit 2 Aeq. Alkohol (73-74 pCt.), als wenn sie mit nur 1 Aeq. (77 pCt.) Alkohol gemengt wird, ein Irrthum ist.

Die Angabe Millon's, dass unter $0^{\circ}$ keine Aetherschwefelsäure gebildet werde, ist auch unrichtig. Die für 


\section{Claesson: Ueber die neutralen u. sauren Sulfate}

diese Behauptung sprechenden Versuche werde ich später mittheilen.

Schliesslich sei erwähnt, dass die Ausbeute von Methylätherschwefelsäure unter ähnlichen Bedingungen ungefähr dieselbe ist, wie von Aethylätherschwefelsäure. Meine nach derselben Methode angestellten Versuche ergaben $59 \mathrm{pCt}$. während für Aethylätherschwefelsäure 54 pCt. gefunden wurden.

\section{V1. Ueber Chlorsulfonsäureäthyläther und seine Einwirkung auf Alkohol.}

Der in dieser Abhandlung bereits erwähnte Aether der Chlorsulfonsäure ist von verschiedenen Chemikern dargestellt worden. Kublmann '), R. Williamson ${ }^{2}$ ) und v. Purgold ${ }^{3}$ ) haben ihn als Hauptprodukt erhalten bei der Einwirkung von Chloräthyl auf Schwefelsäureanhydrid, M. Müller ${ }^{4}$ ) aus Aethylen und Chlorsulfonsäure, und schliesslich Behrend ${ }^{5}$ ) aus Sulfurylchlorid und Alkohol.

Ich habe die Methode Müller's als die einfachste angewendet. Aethylengas, welches vorher Natronlauge und conc. Schwefelsäure passirt hatte, wurde in Chlorsulfonsäure eingeleitet, bis diese damit gesättigt war. Danach wurde das Produkt entweder sogleich im Vacuun destillirt, oder man goss es erst in eiskaltes Wasser und reinigte das dabei abgeschiedene Oel, nachdem es mit wasserfreiem Kupfersulfat getrocknet war, durch Destillation im Vacuum. Die Menge des erhaltenen Oels ist in beiden Fällen dieselbe, sie beträgt etwa die Hälfte des ganzen.

Der Chlorsulfonsäureäthyläther siedet bei $151-154^{\circ}$ (corr.) unter geringer partieller Zersetzung, im Vacuum aber völlig unzersetzt. Der Aether greift äusserst heftig die

1) Ann. Chem. Pharm. 133, 108.

2) Dies. Journ. 73, 73.

3) Zeitschr. Chem. 1868, S. 669.

4) Ber. Berl. chem. Ges. 6, 227.

5) Daselbst 9, 1334. 
Augen an. Nur sein Verhalten zum Alkohol werde ich hier ausführlicher behandeln.

P. Behrend ${ }^{1}$ ) hat hierüber eine Arbeit veröffentlicht, die ich etwas näher beleuchten will. Er stellte Sulfurylchlorid aus Chlorsulfonsäure dar durch Erhitzen in geschlossenen Röhren bis auf $200^{\circ}$ und liess auf die so erhaltene Verbindung Methyl und Aethylalkohol einwirken mit folgendem Resultate.

Bei Einwirkung von Methyl- oder Aethylalkohol (gleiche Moleküle) auf Sulfurylchlorid entstanden der Methyl- oder Aethyläther der Chlorsulfonsäure nebst Chlorwasserstoff nach folgender Gleichung:

$$
\mathrm{SO}_{2} \mathrm{Cl}_{2}+\mathrm{CH}_{3} \mathrm{OH}=\mathrm{CH}_{3} \mathrm{OSO}_{2} \mathrm{Cl}+\mathrm{HCl} \text {. }
$$

Bei Einwirkung von einem weiteren Molekül Alkohol auf die so erhaltenen Aetherarten entstanden die reinen Sulfate nach der Gleichung:

$$
\mathrm{CH}_{3} \mathrm{OSO}_{2} \mathrm{Cl}+\mathrm{C}_{2} \mathrm{H}_{5} \mathrm{OH}=\underset{\mathrm{C}_{2} \mathrm{H}_{5} \mathrm{O}}{\mathrm{CH}_{5} \mathrm{O}} \mathrm{SO}_{2}+\mathrm{HCl} \text {. }
$$

Die so erhaltenen Sulfate stimmten mit den früher erwähnten, von Mazurowska dargestellten überein. Sie wurden augenblicklich von Wasser in Aethersäure und Alkohol zersetzt.

Mazurowska's und Behrend's Untersuchungen sind aus demselben Laboratorium hervorgegangen. Allerdings nehmen sich die Resultate derselben ganz gut auf dem $\mathrm{Pa}$ pier aus, beide sind aber gleich grundfalsch, wie aus den folgenden Versuchen hervorgeht.

Schon M. Müller's Angaben lauten in seiner vortrefflichen, oben citirten Untersuchung über Chlorsulfonsäureäther wörtlich wie folgt:

„Lässt man den Aether in Alkohol tropfen, so findet augenblicklich heftige Erhitzung statt: es geht lebhaft Chloräthyl fort, während Aethylschwefelsäure gebildet wird. Auch reiner Amylalkohol setzt sich damit um zu Chloräthyl und Amyloxydschwefelsäure. Dieser Versuch zeigt, dass das entweichende Chloräthyl aus dem Aether stammt."

I) Ber. Berl. chem. Ges. 9, 1334. 
Diesen Aufsatz scheint Behrend nicht gekannt zu haben, sonst wäre wohl niemals seine Arbeit zu Tage gefördert worden.

Ich kann Müller's Angaben vollständig bestätigen und noch hinzufügen, dass bei der Einwirkung von Chlorsulfonsäureäther auf Alkohol nicht nur die erwähnten Produkte, sondern auch etwas Chlorwasserstoff und viel Aethyläther entstehen. Für die Reaction wurde folgender Apparat angewendet. Ein Kolben, mit einem Scheidetrichter versehen, stand in Verbindung mit einem Liebig'schen Kaliapparat, womit ein mit Eis und Kochsalz abgekühlter Condensationsapparat verbunden war. Der Kolben enthielt absoluten Alkohol, und der Scheidetrichter Chlorsulfonsäureäthyläther. Der Kaliapparat war mit Wasser gefüllt. Die Mischung muss vorsichtig bewerkstelligt werden, sonst tritt durch die gewaltsame Gasentwicklung leicht eine Explosion ein. Der Chlorwasserstoff und ein Theil ron dem gebildeten Aethyläther wurde in dem Kaliapparat, das Uebrige in dem Condensationsapparat verdichtet. Dieses bestand aus einem Gemenge von Chloräthyl und Aether, und siedete zwischen $12^{\circ}$ und $38^{\circ}$ Der Inhalt des Kolbens nach vollzogener Mischung löste sich klar in Wasser und bestand nur aus Aetherschwefelsäure nebst etwas Schwefelsäure und Chlorwasserstoff. Die Reaction geht somit in doppelter Richtung vor sich:

1) $\mathrm{C}_{2} \mathrm{H}_{5} \mathrm{OSO}_{2} \mathrm{Cl}+\mathrm{C}_{2} \mathrm{H}_{5} \mathrm{OH}=\mathrm{C}_{2} \mathrm{H}_{5} \mathrm{Cl}+\mathrm{C}_{2} \mathrm{H}_{5} \mathrm{OSO}_{2} \mathrm{OH}$.

2) $\mathrm{C}_{2} \mathrm{H}_{5} \mathrm{OSO}_{2} \mathrm{Cl}+2 \mathrm{C}_{2} \mathrm{H}_{5} \mathrm{OH}=\left(\mathrm{C}_{2} \mathrm{H}_{5}\right)_{2} \mathrm{O}+\mathrm{HCl}+\mathrm{C}_{2} \mathrm{H}_{5} \mathrm{OSO}_{2} \mathrm{OH}$.

Lässt man umgekehrt Alkohol in den Chlorsulfonsäureäther tropfen, so ist die Gas- und Wärmeentwicklung anfangs gering, steigert sich aber allmählich. Im Anfange entweicht hauptsächlich Chloräthyl, später Chlorwasserstoff. Aethyläther entsteht hierbei nur in geringer Menge. Nach beendeter Operation wurde Wasser zu dem Inhalte des Kolbens gesetzt, wobei ein Oel sich absehied, das sich bei näherer Untersuchung als reines Aethylsulfat herausstellte. Eine dritte Reaction hatte sich somit vollzogen, welche aus folgender Gleichung erhellt:

$$
\mathrm{C}_{2} \mathrm{H}_{5} \mathrm{OH}+\mathrm{C}_{2} \mathrm{H}_{5} \mathrm{OSO}_{2} \mathrm{Cl}=\left(\mathrm{C}_{2} \mathrm{H}_{5} \mathrm{O}\right)_{2} \mathrm{SO}_{2}+\mathrm{HCl} \text {. }
$$


Die Ausbeute an Aethylsulfat ist variabel, aber ungeachtet mehrfacher Modificirung der Versuche habe ich niemals über $20 \mathrm{pCt}$. von der berechneten Menge erhalten.

Es ist leicht zu verstehen, dass in der ersten Reaction mit Aethylalkohol im Ueberschusse kein Aethylsulfat sich gebildet hatte, da Alkohol und Aethylsulfat, wie Erlenmeyer ${ }^{1}$ ) gefunden hat, sich gegenseitig zersetzen:

$$
\left(\mathrm{C}_{2} \mathrm{H}_{5} \mathrm{O}_{2} \mathrm{SO}_{2}+\mathrm{C}_{2} \mathrm{H}_{5} \mathrm{OH}=\left(\mathrm{C}_{2} \mathrm{H}_{5}\right)_{2} \mathrm{O}+\mathrm{HOSO}_{2} \mathrm{OC}_{2} \mathrm{H}_{5}\right. \text {, }
$$

und es kann wohl als wahrscheinlich angesehen werden, dass der bei der Reaction auftretende Aethyläther in dieser Weise entstanden ist.

Behrend hat auch die Einwirkung von Methylalkohol auf Chlorsulfonsäureäthyläther untersucht, wie umgekehrt die Einwirkung von Aethylalkohol auf den Methyläther derselben Säure. In Betreff des ersten Versuchs heisst es:

„In analoger Weise ist es mir gelungen, einen gemischten Aether $\mathrm{SO}_{2}{ }_{\mathrm{OCH}_{3}}^{\mathrm{OCH}_{3} \mathrm{H}_{5}}$ durch Einwirkung von Methylalkohol auf Aethylschwefelsäurechlorid darzustellen. Es ist dies eine schwach gelbliche, sich aber durch Erhitzen leicht dunkler färbende, völlig neutrale Flüssigkeit, die bei der Analyse nachstehende Resultate ergab:

$$
\begin{array}{ccc} 
& \text { Gefunden. } & \text { Berechnet. } \\
\text { C } & 25,55 & 25,71 \\
\text { H } & 5,96 & 5,71
\end{array}
$$

Mit Wasser zerfällt dieser Aether augenblicklich in eine stark saure Flüssigkeit."

Ueber den umgekehrten Versuch: die. Einwirkung von Aethylalkohol auf chlorsulfonsauren Methyläther sagt er:

„Durch Einwirkung von Aethylalkohol auf dieses Methylschwefelsäurechlorid erhält man einen, dem unter I erwähnten gleich zusammengesetzten, neutralen gemischten Aether $\mathrm{SO}_{2} \mathrm{OCH}_{2} \mathrm{OCH}_{5}$, wie folgende Analyse ergiebt:

$\begin{array}{ccc} & \text { Gefunden. } & \text { Berechnet. } \\ \mathrm{C} & 25,56 & 25,71 \\ \mathrm{H} & 5,95 & 5,71\end{array}$

1) Ann. Chem. Pharm. 162, 373. 
252 Claesson: Ueber die neutralen $\mathfrak{u}$. sauren Sulfate

Die nahe liegende Annahme, dass dieser Aether durch Wasser in Methylschwefelsäure und Aethylalkohol zerfallen würde, erwies sich als irrthümlich. Es zerfällt auch dieser Aether in Aethylschwefelsäure und Methylalkohol, so dass wir es hier augenscheinlich nicht mit isomeren, sondern mit identischen Körpern zu thun haben."

Ich habe den ersten dieser Versuche wiederholt, der in derselben Weise abgelaufen ist, wie der früher erwähnte ähnliche. Es gingen Chlormethyl, Chlorwasserstoff und wahrscheinlich anch Methyläther fort, und die rückständige Flüssigkeit bestand hauptsächlich aus Aethylätherschwefelsäure.

Behrend hat bei seinen Versuchen ganz und gar übersehen, dass während der Reaction bedeutende Mengen organischer Substanzen weggehen (nachdem die weggehenden Gase von Salzsäure befreit waren, konnte eine beinahe contimuirliche grosse Flamme damit erzeugt werden), und dass bedeutend mehr als $1 \mathrm{Mol}$. Methylalkohol erforderlich ist, um die vollständige Zersetzung des Chlorsulfonsäureäthers zu bewirken. Nichts desto weniger erhielt Behrend, wie angegeben, bei der Analyse der beiden Produkte Resultate, die mit berechneten auf $2 / 10$ pCt. und mit einander auf $1 / 100$ pCt. nahe übereinstimmen. Dies übersteigt wohl beinahe die Grenzen der Wahrscheinlichkeit, da es ja unmöglich ist, auch wenn das Produkt wirklich das wäre, was Behrend angenommen hat, Salzsäure aus einem in Wasser löslichen Körper vollständig zu entfernen, sowie es auch unmöglich ist, bei einer Reaction mit so heftiger Gasentwicklung die Menge des hinzugesetzten Alkohols so zu berechnen, dass keines von den Agentien im Ueberschusse bleibt.

Es scheint mir hiermit an den Tag gelegt, dass neutrale Sulfate mit den Eigenschaften, welche Mazurowska und Behrend ihnen zuerkennen, in Wirklichkeit nicht existiren, sondern ihren Ursprung haben in der Neigung, vorgefasste Meinungen durch Experimente bestätigt zu finden.

Wie schon erwähnt, giebt Mülle $\mathbf{r}$ folgende Reaction an: $\mathrm{C}_{2} \mathrm{H}_{5} \mathrm{OSO}_{2} \mathrm{Cl}+\mathrm{C}_{5} \mathrm{H}_{11} \mathrm{OH}=\mathrm{C}_{2} \mathrm{H}_{5} \mathrm{Cl}+\mathrm{C}_{5} \mathrm{H}_{11} \mathrm{OSO}_{2} \mathrm{OH}$.

Die Produkte aus der Einwirkung von Methylalkohol auf Chlorsulfonsäıreäthyläther sowohl, wie von Aethylalkohol 
auf chlorsulfonsauren Methyläther geben beide nach Behrend Aethylätherschwefelsäure, somit:

$$
\begin{aligned}
& \mathrm{C}_{2} \mathrm{H}_{5} \mathrm{OSO}_{2} \mathrm{Cl}+\mathrm{CH}_{3} \mathrm{OH}=\mathrm{C}_{2} \mathrm{H}_{5} \mathrm{OSO} \mathrm{SO}_{2} \mathrm{OH}+\mathrm{CH}_{3} \mathrm{Cl} . \\
& \mathrm{CH}_{3} \mathrm{OSO}_{2} \mathrm{Cl}+\mathrm{C}_{2} \mathrm{H}_{5} \mathrm{OH}=\mathrm{C}_{2} \mathrm{H}_{5} \mathrm{OSO} \mathrm{O}_{2} \mathrm{OH}+\mathrm{CH}_{3} \mathrm{Cl} .
\end{aligned}
$$

Hieraus scheint als Regel hervorzugehen, dass wenn ein Alkohol auf einen Aether der Chlorsulfonsäure wirkt, das Radical mit der höchsten Zahl von Kohlenatomen in dem Produkt als Aetherschwefelsäure enthalten bleibt, das mit der niedrigeren Kohlenstoffatomzahl aber mit Chlor in Verbindung tritt.

\section{Ueber eine neue Bildungsweise der Aethionsäure.}

Im vorigen Abschnitte habe ich erwähnt, dass das Produkt der Einwirkung von Aethylen auf Chlorsulfonsäure nur etwa zur Hälfte aus Chlorsulfonsäureäthyläther besteht. Nachdem der Chlorsulfonsäureäther überdestillirt ist, bleibt nämlich in der Retorte bei allen Versuchen, die ich angestellt habe, ein bedeutender schwarz gefärbter Rückstand. Um zu erforschen, woraus dieser bestehe, wurde er in Wasser gelöst, mit kohlensaurem Baryt neutralisirt und die filtrirte Lösung mit Thierkohle entfärbt, worauf Alkohol zugesetzt wurde, bis ein dauernder Niederschlag entstand." Der grösste Theil des Salzes krystallisirte dann nach einiger Zeit in kleinen weissen nierenförmigen Krystallmassen. Diese wurden in Wasser wieder gelöst, mit Alkohol gefällt, und diese Operation so lange wiederholt, bis das Produkt chlorfrei war, wonach das Salz über Schwefelsäure getrocknet und analysirt wurde. Die Resultate der Analysen sowohl, als die Eigenschaft der Lösung des Salzes, beim Kochen durch Ausscheidung von schwefelsaurem Baryt getrübt zu werden, und schliesslich vollständig in schwefelsauren Baryt und Isäthionsäure überzugehen, zeigte, dass es äthionsaurer Baryt war.

1) 0,533 Grm. gaben 0,357 Grm. $\mathrm{Ba} \mathrm{SO}_{4}$.

2) 0,505 Grm. gaben $0,640 \mathrm{Grm} . \mathrm{Ba} \mathrm{SO}_{4}$ (Schwefelbest.).

3) 0,4205 Grm. gaben $0,114 \mathrm{Grm}$. $\mathrm{CO}_{2}$ und 0,062 Grm. $\mathrm{H}_{2} \mathrm{O}$. 
254 Claesson: Ueber die neutralen u. sauren Sulfate

Berechnet. Gefunden.

\begin{tabular}{lrrr}
$\mathrm{Ba}$ & 137 & 39,14 & 39,38 \\
$\mathrm{O}_{71 / 3}$ & 120 & 34,30 & - \\
$\mathrm{S}_{2}$ & 64 & 18,29 & 17,40 \\
$\mathrm{C}_{2}$ & 24 & 6,85 & $\mathbf{7 , 3 9}$ \\
$\mathrm{H}_{5}$ & 5 & 1,42 & 1,63 \\
\cline { 2 - 3 } & 350 & 100,00 &
\end{tabular}

Der Berechnung ist die von Marchand angegebene Formel $\mathrm{C}_{2} \mathrm{H}_{4} \backslash \mathrm{SO}_{2} \mathrm{O} O \mathrm{Oa}+1 / 2$ aq. zu Grunde gelegt. Die Resultate der Analyse passen aber eben. so gut auf ein wasserfreies Salz.

Das Rohmaterial für die Darstellung des Chlorsulfonsäureäthers enthält somit in ziemlich grossen Mengen ein Nebenprodukt, aus welchem ohne grosse Mühe reine äthionsaure Salze dargestellt werden können. Auch für die Darstellung der Isäthionsäure ist diese Methode der bisher gebräuchlichen aus Alkohol oder Aether und Schwefelsäure weit vorzuziehen.

Es erhebt sich nun die Frage, wie diese Aethionsäure entstanden ist.

Sowohl Müller ${ }^{1}$ ), wie v. Purgold ${ }^{2}$ ) haben wahrgenommen, dass bei Einwirkung von Chloräthyl auf Schwefelsäureanhydrid neben dem Chlorsulfonsäureäthyläther ein in Wasser löslicher Körper entsteht, welcher mit Wasser verdünnt, gekocht und mit kohlensaurem Barium neutralisirt, nach Müller isäthionsaures Barium, nach v. Purgold ein Gemenge von diesem Salz und chloräthylsulfonsaurem Barium giebt. Dieselben Resultate wurden erhalten bei Sättigung von chlorsulfonsaurem Aethyläther mit Schwefelsäureanhydrid. Hieraus schliesst Müller, dass der chlorsulfonsaure Aethyläther das primäre Produkt ist, v. Purgold glaubt aber, dass dabei die isomeren Verbindungen $\mathrm{C}_{2} \mathrm{H}_{5} \mathrm{OSO}_{2} \mathrm{Cl}$, $\mathrm{C}_{2} \mathrm{H}_{4} \mathrm{ClSO}_{2} \mathrm{OH}$ und $\mathrm{C}_{2} \mathrm{H}_{4} \mathrm{OHSO}_{2} \mathrm{Cl}$ gleichzeitig entstehen. v. Purgold's Annahme, namentlich dass Isäthionsäure oder

1) Ber. Berl. chem. Ges. 6, 227.

2) Daselbst 6, 502. 
dessen Chlorid bei dieser Reaction direct entstehen soll, kann bestimmt nicht richtig sein, da Isäthionsäure mit Schwefelsäureanhydrid Aethionsäure bilden muss. Keiner von Beiden kam auf den Gedanken, dass die Isäthionsäure durch Zersetzung der in erster Hand gebildeten Aethionsäure entstanden sei. Dass aber dies wirklich der Fall ist, liegt auf der Hand. Man hat somit die folgenden Reactionen:

1) $\mathrm{C}_{2} \mathrm{H}_{5} \mathrm{CI}+\mathrm{SO}_{3}=\mathrm{C}_{2} \mathrm{H}_{5} O \mathrm{SO}_{2} \mathrm{Cl}$,

2). $\mathrm{C}_{2} \mathrm{H}_{5} \mathrm{OSO}_{2} \mathrm{Cl}+\mathrm{SO}_{3}=\mathrm{C}_{2} \mathrm{H}_{4} \stackrel{\mathrm{SO}_{2} \mathrm{OH}}{\mathrm{OSO}_{2} \mathrm{Cl}}$;

dazu kommt nach v. Purgold die Reaction:

$$
\mathrm{C}_{2} \mathrm{H}_{5} \mathrm{Cl}+\mathrm{SO}_{3}=\mathrm{C}_{2} \mathrm{H}_{1} \mathrm{ClSO}_{2} \mathrm{OH} \text {. }
$$

Die Annahme liegt nahe, dass die bei der Einwirkung von Aethylen auf Chlorsulfonsäure entstandene Aethionsäure eigentlich durch Einwirkung von Chlorsulfonsäure auf den zuerst gebildeten Aether dieser Säure entstanden ist, wie folgende Gleichung versinnlicht:

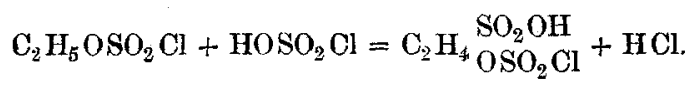

Directe Versuche zeigten nun, dass der Chlorsulfonsäureäther bei gewöhnlicher Temperatur wenig von der Chlorsulfonsäure angegriffen wird, dass aber nach Einwirkung bei $100^{\circ}$ das Produkt grösstentheils in Wasser löslich war, und dass die Lösung Aethionsäure enthielt.

Es ist demnach wohl richtig, anzunehmen, dass sowohl bei Einwirkung von Schwefelsäureanhydrid auf Chloräthyl, wie von Aethylen auf Chlorsulfonsäure erst Chlorsulfonsäureäthyläther entsteht, der durch Ueberschuss von Anhydrid oder Chlorsulfonsäure gänzlich oder theilweise in Aethionsäure übergehen kann.

\section{Ueber Aethylsulfat.}

Wie bekannt, stellte Wetherill ${ }^{1}$ ) in Liebig's Laboratorium durch Einleiten der Dämpfe von Schwefelsäure-

1) Aun. Chem. Pharm. 66, 117. 
anhydrid in Aether oder Alkohol einen Aether mit folgenden Eigenschaften dar.

Bei vorsichtiger Destillation desselben gingen bei einer Temperatur von $110-120^{\circ}$ Oeltropfen über, aber beim Erhöhen der Temperatur bis zu $130^{\circ}-140^{\circ}$ trat vollständige Zersetzung ein. Das geringe Destillat, welches erhalten wurde, hatte dieselbe procentische Zusammensetzung, wie das undestillirbare Oel. Beide gaben bei der Analyse die für das Aethylsulfat berechneten Werthe. Beim Kochen mit Wasser löste sich der Aether schnell auf, und die so erhaltene Lösung enthielt ausser Alkohol und Schwefelsäure drei Säuren, nämlich kleine Mengen von Methionsäure nebst Aethylätherschwefelsäure und Isäthionsäure, die beiden letzteren zu gleichen Theilen. Wie bekannt, wurde der Aether als das neutrale Sulfat der Schwefelsäure betrachtet.

Erlenmeyer ${ }^{1}$ ) hat die Versuche $W$ etherill's wiederholt und fand $6,47 \mathrm{pCt}$. Isäthionsäure, aus welchem Umstande er schliesst, dass dieser Aether ein Gemenge von Aethylsulfat und isäthionsaurem Aethyläther sei. Erlenmeyer zeigte auch, dass bei der Einwirkung dieses Aethers auf Alkohol Aethyloxyd entstehe.

Baumstark erhielt, wie schon erwähnt, bei der Einwirkung von Pyrosulfurylchlorid auf Alkohol und Aether ein Oel mit ähnlichen Eigenschaften, wie die Aether Wethe rill's.

Die neutralen Sulfate von Orlowsky-Mazurowska und von Behrend habe ich schon ausführlich behandelt und deren Angaben widerlegt.

Schliesslich hat neulich lant einer Correspondenzmittheilung in den Berliner Berichten aus Petersburg Stempnewsky ${ }^{2}$ ) aus Silbersulfat und Jodäthyl einen Aether dargestellt, der in seinen Eigenschaften "durchaus von denjenigen des von Fräulein Mazurowska bereiteten Esters verschieden ist". Mehr enthält die Mittheilung nicht.

1) Ann. Chem. Pharm. 162, 382.

2) Ber. Berl. chem. Ges. 11, 514. 
Aus diesen Angaben geht hervor, dass Aethylsulfat ein höchst unvollständig gekannter Körper ist.

Ich werde hier die Darstellung und Eigenschaften des reinen Aethylsulfats besprechen und zugleich eine vergleichende Untersuchung anstellen zwischen dem reinen Aethylsulfat, Wetherill's Verbindung und dem seit Mitte des vergangenen Jahrhunderts gekannten sogenannten schweren Weinöl.

Ich habe schon erwähnt, dass bei Einwirkung von Alkohol auf Chlorsulfonsäureäther unter anderen Produkten Aethylsulfat entsteht nach der Reaction:

$$
\mathrm{C}_{2} \mathrm{H}_{5} \mathrm{OSO}_{2} \mathrm{Cl}+\mathrm{C}_{2} \mathrm{H}_{5} \mathrm{OH}=\left(\mathrm{C}_{2} \mathrm{H}_{5} \mathrm{O}\right)_{2} \mathrm{SO}_{2}+\mathrm{HCl} \text {. }
$$

Aus der Mischung kann das Sulfat mit Wasser als Oel gefällt werden, welches, in üblicher Weise getrocknet, durch Destillation im Vacuum rein erhalten wird.

Auch kann es durch vorsichtige Destillation von wassertreier Aetherschwefelsäure im Vacuum bei etwa $140^{\circ}$ gewonnen werden. Die Ausbeute ist hier aber gering, was auf folgenden Umständen beruht. Die Aethylätherschwefelsäure wird in Aethylsulfat und Schwefelsäure dissociirt, bei einer unter dem Siedepunkt des Aethylsulfats liegenden Temperatur. Wie später gezeigt wird, fängt diese Dissociation schon bei $100^{\circ}$ an. Bei einer Temperatur von $140^{\circ}$ muss sie daher weit fortgeschritten, wenn nicht ganz vollständig sein. Das Aethylsulfat siedet aber unter gewöhnlichem Luftdruck erst bei $208^{\circ}$. Weit unter dieser Temperatur wird das Aethylsulfat von der gleichfalls gebildeten Schwefelsäure iu schweflige Säure und humusähnliche Substanzen zersetzt.

Das Aethylsulfat kann noch auf eine dritte Weise dargestellt werden. Wird ein Gemenge von gleichen Molekülen absolutem Alkohol und Schwefelsäure nach dem Erkalten erst mit Eis und darauf mit Wasser verdünnt und die so erhaltene Flüssigkeit mit Chloroform extrahirt, so nimmt dieses das Aethylsulfat aus der Lösung auf. Aus einer Mischung von $400 \mathrm{Grm}$. Alkohol und einem entsprechenden Gewicht Schwefelsäure wurden durch eine solche Extraction $25 \mathrm{Grm}$. reines Aethylsulfat gewonnen, nach Siedepunkt und übrigen 


\section{Claesson: Ueber die neutralen u. sauren Sulfate}

Eigenschaften den früher erwähnten völlig ähnlich. Dies zeigt, dass schon bei der Temperatur, welche beim Mischen von Alkohol und Schwefelsäure entsteht, die gebildete Aethersäure zum kleinen Theil in Aethylsulfat und Schwefelsäure dissociirt wird.

Ich habe auch die Einwirkung von Silbersulfat auf Aethyljodid untersucht. Beide wurden in berechneten Mengen und in völlig trocknem Zustande zugleich mit etwas Alkohol und wasserfreiem Aether in Röhren eingeschlossen, um sodann 3 Stunden auf $150^{\circ}$ erhitzt zu werden. Dabei setzen sich die Substanzen völlig quantitativ um nach der Gleichung: $\mathrm{Ag}_{2} \mathrm{SO}_{4}+2 \mathrm{C}_{2} \mathrm{H}_{5} \mathrm{~J}=\left(\mathrm{C}_{2} \mathrm{H}_{5}\right)_{2} \mathrm{SO}_{4}+2 \mathrm{Ag} \mathrm{J}$. Das auf diese Weise gewonnene Aethylsulfat stimmt in Siedepunkt, spec. Gew. und übrigen Eigenschaften völlig mit dem vorher beschriebenen überein.

Aethylsulfat ist in seinen Eigenschaften dem Methylsulfat sehr ähnlich. Es ist ein farbloses, in Wasser unlösliches Oel von sehr angenehmem, etwas an Pfeffermünze erinnerndem Geruch, welches unter gewöhnlichem Luftdruck bei $208^{\circ}$ (Quecksilbersäule ganz in Dampf) unter geringer partieller Zersetzung siedet. Im Vacuum destillirt es unzersetzt bis auf den letzten Tropfen über. Spec. Gew. bei $19^{\circ}$ 1,1837. Bei der Analyse wurden folgende Zahlen erhalten:

0,387 Grm. gaben 0,440 Grm. $\mathrm{CO}_{2}$ und 0,231 Grm. $\mathrm{H}_{2} \mathrm{O}$.

$0,321 \mathrm{Grm}$. gaben 0,480 Grm. $\mathrm{Ba} \mathrm{SO}_{4}$.

$\begin{array}{lccc} & & \text { Berechnet. } & \text { Erhalten. } \\ \mathrm{C}_{4} & 48 & 31,17 & 31,01 \\ \mathrm{H}_{10} & 10 & 6,49 & 6,63 \\ \mathrm{~S} & 32 & 20,78 & 20,53 \\ \mathrm{O}_{4} & 64 & 41,56 & -\end{array}$

Das Gasvolumgewicht ist durch den folgenden Versuch nach Hofmann's Methode ermittelt:

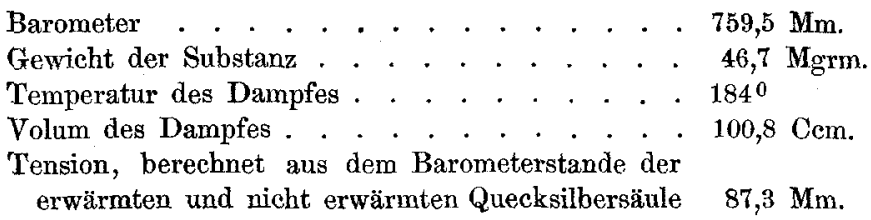


Hieraus ergiebt sich die Dampfđichte zu 75,3, während der Formel $\mathrm{C}_{4} \mathrm{H}_{10} \mathrm{SO}_{4} 77$ entspricht. Die Differenz dürtte nicht ganz von Beobachtungsfehlern, sondern zum Theil von geringer Zersetzung der vergasten Substanz herrühren, was daraus hervorgeht, dass die T'ension der Substanz nach dem Versuche $2 \mathrm{Mm}$. grösser, als vor dem Versuche war.

Ist das Aethylsulfat längere Zeit mit Wasser in Berührung, so nimmt dieses eine saure Reaction an, doch geht die Zersetzung äusserst langsam vor sich. Ein paar Tropfen können damit Monate lang in Berührung sein, ohne dass deren Volum merkbar vermindert wird. Beim Kochen mit Wasser wird es je nach der Menge in kürzerer oder längerer Zeit zersetzt, aber die Einwirkung ist viel langsamer, als bei dem entsprechenden Methyläther. Die Zersetzung äussert sich zuerst in der Bildung von Alkohol' und Aetherschwefelsäure, die ihrerseits; wie bekannt, in Alkohol und Schwefelsäure zerlegt wird.

Mit Alkohol setzt sich der Aether beim Erwärmen in Aethyläther und Aethylätherschwefelsäure um nach der Gleichung:

$$
\left(\mathrm{C}_{2} \mathrm{H}_{5} \mathrm{O}\right)_{2} \mathrm{SO}_{2}+\mathrm{C}_{2} \mathrm{H}_{5} \mathrm{OH}=\mathrm{C}_{2} \mathrm{H}_{5} \mathrm{OSO} \mathrm{O}_{2} \mathrm{OH}+\left(\mathrm{C}_{2} \mathrm{H}_{5}\right)_{2} \mathrm{O} .
$$

Ich habe auch Wetherill's Aethylsulfat durch Einleiten von Dämpfen von Schwefelsäureanhydrid in Aether dargestellt. Bei der Destillation verhielt es sich, wie Wetherill angegeben hat. Nur ein sehr geringer Theil destillirte über, das Uebrige wurde zersetzt. Nach der Zersetzung des Oels durch längeres Kochen mit Wasser wurden 12 pCt. Isäthionsäure gefunden. Aus dem ersten Versuche scheint hervorzugehen, dass das Oel nur zum kleinen Theil aus Aethylsulfat bestand, aus dem letzten, dass das Sulfat den grössten Theil ausmachte. Ein anderer Theil des Oels wurde daher einer Destillation im Vacuum unterworfen, wobei es sich zeigte, dass es bis auf etwa $1 / 8$ überging, und dass das Destillat alle Eigenschaften des Aethylsulfats hatte. Der in der Retorte gebliebene Rückstand wurde unmittelbar, nachdem das Aethylsulfat überdestillirt war, zersetzt. Die Erklärung, dass so wenig bei gewöhnlicher Temperatur überdestillirt, ist somit darin zu suchen, dass der in dem Oel 
enthaltene Aether der Isäthionsäure schon unter der Siedetemperatur des Sulfats zersetzt wird, und die dabei entstehenden Produkte auf das Sulfat selbst zerlegend einwirken.

Ich will schliesslich erwähnen, dass die Ausbeute an Aethylsulfat nach Wetherill's Methode, wenn wasser- und alkoholfreier Aether stets in grossem Ueberschusse angewendet wird, so gross ist, dass dies zur Annahme berechtigt, Aethylsulfat sei das primäre Produkt der Einwirkung von Schwefelsäureanhydrid auf Aether.

Im vorigen und im Anfange dieses Jahrhunderts stellte man den gewönnlichen Aether durch Erhitzen von starkem Spiritus und Vitriolöl dar. Die Operation wurde in einer Retorte vorgenommen, welche im Sandbade erhitzt wurde. Was zuerst überging, wurde besonders aufgefangen und Aether genannt. Dann wurde die Vorlage gewechselt und die Destillation fortgesetzt, wobei ein aus drei Schichten bestehendes Destillat resultirte, von welchen die oberste und unterste eine ölartige Beschaffenheit hatten und Oleum vitrioli dulce genannt wurden. ${ }^{1}$ )

Diese Nebenprodulkte der Aetherbereitung, auch bei trockner Destillation der ätherschwefelsauren Salze entstehend, waren zu wiederholten Malen Gegenstand umafassender Untersuchungen von Hennel, Dumas und Boullay, Serullas, Marchand und Liebig. Hier mag nur in aller Kürze erwähnt werden, dass man aus dem Rohprodukt, welches später schweres Weinöl genannt wurde, durch Einwirkung von Alkalien oder Behandeln mit siedendem Wasser zwei neue Körper isolirte, einen flüssigen, genannt A ether ol, und einen festen, Aetherin. Diese sind vielfach in der Literatur und von den damaligen Gelehrten mit dem schweren Weinöl verwechselt worden. Sie sind zusammen auch leichtes Weinöl genannt. Sie haben nach Hennel's, Serullas' und Marchand's Analysen die procentische Zusammensetzung des Aethylens. Das schwere Weinöl selbst wurde nach Berzelius und Liebig, Hennel's Analysen zufolge, als

1) Vergl. Malouin, Chimie Medicinale, Paris 1755. 
schwefelsaures Aethyloxyd-Aetherol: $\left(\mathrm{C}_{2} \mathrm{H}_{5} \mathrm{O}_{2} \mathrm{SO}_{2}+\mathrm{C}_{4} \mathrm{H}_{8} \mathrm{SO}_{3}\right.$ angesehen.

Es braucht kaum gesagt $z \mathfrak{u}$ werden, dass das schwere Weinöl hauptsächlich aus Aethylsulfat besteht. Ich habe es durch trockne Destillation von ätherschwefelsaurem Kali allein oder mit gebranntem Kalk dargestellt, wobei freilich nur sehr kleine Mengen eines Oels erhalten wurden, welches, durch Destillation im Vacuum gereinigt, sich als reines Aethylsulfat herausstellte. Das Aetherol oder Aetherin ist offenbar ein ganz zufälliger Bestandtheil davon. Erlenmeyer giebt auch an, dass das durch Erhitzen eines Aethergemisches erhaltene schwere Weinöl sich meistens beim Zersetzen mit Wasser klar löste und somit kein leichtes Weinöl enthielt.

\section{Ueber Gerhardt's Parathionsäure.}

Gerhardt hat in seinem Traité de Chimie organique einer mit der Aetherschwefelsäure isomeren Säure erwähnt. Er sagt, dass eine Lösung von ätherschwefelsaurem Baryt beim Kochen sauer werde und schwefelsauren Baryt absetze. Wenn dieser abfiltrirt und das Filtrat mit kohlensaurem Baryt gesättigt wird, so wird die Lösung nachher beim Sieden nicht zersetzt. Er schliesst hieraus das Vorhandensein von zwei isomeren Aethersäuren, von welchen or die eine Parathionsäure nennt.

Die Identität dieser beiden Säuren sucht Erlenme y er ${ }^{1}$ ) nachzuweisen, indem er zeigt, dass eine erhitzte und nachher neutralisirte Lösung von ätherschwefelsaurem Baryt beim Kochen zersetzt wird, und zwar um so mehr, je stärker die Lösung concentrirt war.

Dass eine Lösung von ätherschwefelsaurem Baryt, sowie im Allgemeinen Lösungen von Salzen dieser Säure in concentrirtem Zustande auch ohne Erwärmen langsam zersetzt werden, ist allen denen ein bekanntes Factum, welche krystallisirte Saize dieser Säure hergestellt haben, die Lö-

1) Ann. Chem. Pharm. 162, 382. 


\section{Claesson: Ueber die neutralen u. sauren Sulfate}

sungen mögen nun vorher gekocht werden oder nicht. Eine solche absolute Zersetzbarkeit hat auch Gerhardt sicherlich nicht gemeint.

Aber es giebt eine andere Zersetzung, die leicht wahrgenommen werden kann. Nach vorsichtigem Verdünnen einer Mischung von gleichen Molekülen Alkohol und Schwefelsäure, Neutralisiren mit kohlensaurem Baryt, Filtriren und Erwärmen der so erhaltenen Lösung setzt sich schon vor $100^{\circ}$ ein ganz bedeutender Niederschlag von schwefelsaurem Baryt ab. Wird die Lösung nun neutralisirt und filtrirt, so entsteht bei erneuerter Erwärmung dieser Niederschlag nicht. Ich glaube, dass dieses Gerhardt zur Annahme einer isomeren Säure veranlasst hat, aber es kann gleichwohl in einer Verunreinigung des Salzes mit einem anderen Körper seinen Grund haben, und die bereits erwähnte Beobachtung, dass das Gemisch von Alkohol nnd Schwefelsäure eine kleine Menge von Aethylsulfat enthält, erklärt vollständig die genannte Eigenschaft des ätherschwefelsauren Baryts, beim Erwärmen schwefelsauren Baryt auszuscheiden. Beim Erwärmen zersetzt sich nämlich das Aethylsulfat mit Wasser, wobei schliesslich Schwefelsäure gebildet wird.

Hieraus geht klar hervor, dass Gerhardt's Parathionsäure nicht existirt.

Erlenmeyer ${ }^{1}$ ) giebt als wahrscheinliche Ursache des

1) In Erlenmeyer's Aufsatz kommt eine analytische Angabe von Dr. Janke vor, über die Bestimmung des Wassergehalts von krystallisirtem ätherschwefelsaurem Baryt. Es heisst daselbst ohne Weiteres :

,2,4614 Grm. dieses Salzes verloren über Schwefelsäure 0,208 Grm. Wasser $=8,45 \%$; für einen Gehalt von $2 \mathrm{Mol}$. berechnen sich $8,51 \%$."

Ich will hierzu nur bemerken, dass der Wassergehalt des Barytsalzes eben die Achillesferse der hervorragendsten Chemiker gewesen ist bei allen Versuchen, die Zusammensetzung der Aetherschwefelsäure zu bestimmen. Nach Aufwendung von vieler Mühe stellte es sich heraus, dass es unmöglich war, das Salz unzersetzt in wasserfreiem Zustande zu erhalten. Mir ist es auch nicht gelungen, das Salz wasserfrei rein $\mathbf{z u}$ erhalten, weder durch Erwärmen, noch durch Trocknen im 
Zersetzens einer Lösung von ätherschwefelsaurem Baryt Folgendes an:

„Ich halte es für möglich, dass durch die Einwirkung des Wassers der äthylschwefelsaure Baryt in erster Linie nach folgender Gleichung zersetzt wird:

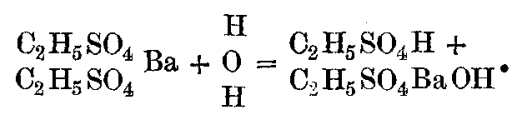

Die freie Aethylschwefelsäure zerfällt dann ziemlich leicht unter Aufnahme eines Moleküls Wasser in Alkohol und Schwefelsäure. Es kann freilich auch sein, dass das $\mathrm{Salz} \mathrm{C}_{2} \mathrm{H}_{5} \mathrm{SO}_{4} \mathrm{BaOH}$ geradeauf in Alkohol und schwefelsauren Baryt zerfällt."

Erlenmeyer nimmt mit anderen Worten an, dass das neutrale Sulfat primär in basisches Salz und freie Säure zersetzt werde. Mir scheint dieses aber eine gesuchte und jeden Grundes entbehrende Erklärung zu sein. So viel ich weiss, kann die Chemie kein einziges wirklich basisches Barytsalz aufweisen. Die Fähigkeit, basische Salze zu bilden, kommt bekanntlich nur schwachen Basen zu. Wie wollte Erlenmeyer z. B. die Zersetzung des Natronsalzes erklären? Auch dieses Salz wird nämlich in concentrirter Lösung etwas zersetzt. Ein basisches Natronsalz mit einer so starken Säure, wie die Aetherschwefelsäure, wäre eine noch grössere Ungereimtheit.

Die wahrscheinlichste und einfachste Annahme ist wohl

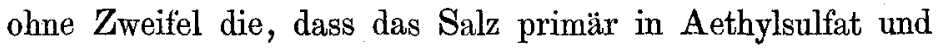
schwefelsauren Baryt zersetzt werde. Die Säure selbst ist ja einer solchen Zersetzung leicht unterworfen.

Folgendes ist eine kurze Zusammenstellung der allgemeinen Resultate, welche sich aus den im Vorhergehenden mitgetheilten Untersuchungen ergeben:

Exsiccator. Es bildet sich dabei schwefelsaurer Baryt, freie Schwefelsäure und Alkohol oder Aether. Wäre dieses nun nach Dr. Janke völlig irrig, so sollte man wenigstens erwarten, die Fehler der Vorgänger erwähnt zu finden. 


\section{Claesson: Ueber die neutralen u. sauren Sulfate}

Die Untersuchungen von Baumstark und OrlowskyMazurowska über die Einwirkung der einsäurigen Alkohole auf die Chlorsulfonsäure sind nicht richtig, sei es aus dem Grunde, dass die angewendete Säure keine Chlorsulfonsäure war, oder dass die Deutung der erhaltenen Resultate falsch ist.

Methyl- und Aethylalkohol reagiren auf Chlorsulfonsäure hauptsächlich nach der Gleichung: $\mathrm{ROH}+\mathrm{HOSO}_{2} \mathrm{Cl}$ $=\mathrm{ROSO}_{2} \mathrm{OH}+\mathrm{HCl}$. Ausserdem bilden sich, je nach den Verhältnissen, verschiedene Mengen von Aetherarten der Chlorsulfonsäure. Die so gewonnenen wasserfreien Aethersäuren sind ölartige Flüssigkeiten, welche in Aether löslich sind, welche Eigenschaft jedoch durch Zusatz von $W$ asser in Folge molecularer Aufnahme von Wasser verloren geht.

Die neutralen Sulfate des Methyl- und Aethylalkohols entstehen durch Dissociation der entsprechenden sauren Sulfate in freie Schwefelsäure und neutrales Sulfat. - Aethylsulfat entsteht auch durch Vereinigung von Aether und Schwefelsäureanhydrid, ferner durch Einwirkung von Alkohol auf Chlorsulfonsäureäthyläther, und von Silbersulfat auf Jodäthyl bei $150^{\circ}$. Die so dargestellten Sulfate sind wasserhelle, in Wasser unlösliche Flüssigkeiten von angenehmem Geruch. Aethylsulfat siedet bei $208^{\circ}$, Methylsulfat bei $187^{\circ}$, beide unter theilweiser Zersetzung, im Vacuum aber völlig unverändert. Durch die Einwirkung von Wasser oder Alkohol bei höheren Temperaturen werden sie wieder in saure Sulfate zersetzt, unter gleichzeitiger Entstehung von Alkohol oder Aether.

Dumas' Methylsulfat, Wetherill's Aethylsulfat und das schwere Weinöl sind mehr oder weniger verunreinigte neutrale Sulfate.

Beim Vermischen von Methyl- oder Aethylalkohol mit Schwefelsäurehydrat werden unter Anwendung gleicher Moleküle 57-59 pCt. Aethersäure gebildet. Diese Ausbeute wird aber erhöht durch weiteren Zusatz entweder von Alkohol oder von Schwefelsäure. Unter Anwendung von $3 \mathrm{Mol}$. Alkohol auf 1 Mol. Schwefelsäure ist das Maximum, 77,4 
pCt., erreicht. Nebenbei entstehen kleine Mengen neutrales Sulfat.

Gerhard t's Parathionsäure existirt nicht; seine Beobachtung ist durch einen Gehalt des ätherschwefelsauren Baryts an neutralem Aethylsulfat veranlasst.

Durch Einwirkung von Aethylengas auf Chlorsulfonsäure entstehen gleichzeitig der Aethyläther dieser Säure und Aethionsäure.

Behren d's Angaben über die Einwirkung von Alkoholen auf Chlorsulfonsäureäther sind nicht richtig. Diese Substanzen reagiren, je nach den Verbältnissen, nach folgenden Gleichungen auf einander:

1) $\mathrm{C}_{2} \mathrm{H}_{5} \mathrm{OSO}_{2} \mathrm{Cl}+\mathrm{C}_{2} \mathrm{H}_{5} \mathrm{OH}=\mathrm{C}_{2} \mathrm{H}_{5} \mathrm{Cl}+\mathrm{C}_{2} \mathrm{H}_{5} \mathrm{OSO}_{2} \mathrm{OH}$.

2) $\mathrm{C}_{2} \mathrm{H}_{5} \mathrm{OSO}_{2} \mathrm{Cl}+2 \mathrm{C}_{2} \mathrm{H}_{5} \mathrm{OH}=\left(\mathrm{C}_{2} \mathrm{H}_{5}\right)_{2} \mathrm{O}+\mathrm{HCl}+\mathrm{C}_{2} \mathrm{H}_{5} \mathrm{OSO}_{2} \mathrm{OH}$.

3) $\mathrm{C}_{2} \mathrm{H}_{5} \mathrm{OSO}_{2} \mathrm{Cl}+\mathrm{C}_{2} \mathrm{H}_{5} \mathrm{OH}=\left(\mathrm{C}_{2} \mathrm{H}_{5} \mathrm{O}_{2} \mathrm{SO}_{2}+\mathrm{HCl}\right.$.

Dabei ist zu bemerken, dass der Aethyläther auch entstanden sein kann durch Einwirkung von Alkohol auf das Aethylsulfat. Enthält der Alkohol und der Chlorsulfonsäureäther verschiedene Alkoholradicale, so findet sich in dem Produkte der Alkohol mit der höchsten Zahl von Kohlenatomen als Aethersäure, der mit der niedrigsten wird in Chlorid umgewandelt.

Die Ursache der Zersetzung von ätherschwefelsauren Salzen im feuchten Zustande liegt wahrscheinlich darin, dass diese, ähnlich wie die freien Aethersäuren, zuerst in neutrale Sulfate des Metalls und des Alkohoiradicals zerfallen.

Lund, März 1879. 